\title{
Il mito prima del mito. Sulla poesia di Cesare Pavese
}

\author{
FAUSTO CURI \\ Università degli Studi di Bologna \\ fcuri@alma.unibo.it
}

\section{Riassunto}

Il saggio, alla luce della grande capacità critica dello stesso Cesare Pavese, analizza la sua raccolta poetica del '36: Lavorare stanca, allo scopo di dimostrare la straordinaria originalità della poesia dello scrittore piemontese. La concezione pavesiana della poesia-racconto $\mathrm{e}$ dell'immagine oggettiva fanno della sua scrittura poetica un unicum all'interno del panorama lirico italiano degli anni ' 30 .

Parole chiave: Poesia pavesiana, ermetismo, originalità oggettiva, realismo narrativo, Pavese, Lavorare stanca.

The myth prior to the myth. About Cesare Pavese's poetry

\begin{abstract}
The essay analyzes the collection of poetry of 1936: Lavorare stanca, through the large critical ability of Cesare Pavese, for demonstrating the extraordinary originality of his own poetry. The pavesian conception of «poesia-racconto» and the objective image converts his poectical writing in a unicum inside the Italian lirical panorama of the thirties.
\end{abstract}

Key words: Pavesian poetry, ermetismo, objective originality, narrative realism, Pavese, Lavorare stanca.

Curi, Fausto. 2011. Il mito prima del mito. Sulla poesia di Cesare Pavese. Cuadernos de Filología Italiana, $\mathrm{n}^{\circ}$ extraordinario: págs. 131-144.

Procediamo, per il momento, un po' didascalicamente, ricordando fatti per forza di cose abbastanza noti. Cesare Pavese giunge relativamente tardi all'elaborazione di una vera e propria teoria del mito. È nel settembre del 1943, quando lo scrittore ha trentacinque anni, che, nel Mestiere di vivere, troviamo le prime annotazioni 
riguardanti il mito. Pavese ha già alle spalle Ciao Masino e Lavorare stanca, oltre a varie prove giovanili e extravaganti sia in verso sia in prosa. Le annotazioni del diario verranno rielaborate e sviluppate e diverranno il saggio Del mito, del simbolo e d'altro, pubblicato dalla rivista «Terraferma» il 10 novembre 1945. Insieme con i saggi Stato di grazia e Adolescenza esso verrà compreso in Feria d'agosto, anch'esso del 1945, dove i tre scritti costituiscono una sorta di trilogia estetico-antropologica alla quale Pavese ha affidato le sue idee sul mito e sulla poesia.

Abbastanza precoci sono invece le prime riflessioni sulla propria attività poetica, giacché si legano alla prima pubblicazione di Lavorare stanca e hanno per titolo Il mestiere di poeta, del 1934, cui seguirà, nel 1940, lo studio A proposito di certe poesie non ancora scritte. L'indagine intorno alla poesia precede quindi quella sul mito, ma è solo quando si troverà a riflettere sul mito che Pavese troverà un assetto teorico definitivo per l'attività poetica.

Non indugerò a riassumere i punti principali di tutte queste riflessioni, che sono ben noti agli studiosi di Pavese. Ciò che mi sembra importante è fermare l'attenzione su alcuni aspetti particolarmente significativi delle teorie pavesiane.

Conviene segnalare, in primo luogo, l'inquietudine teorica di questo scrittore, il bisogno, cosi frequente e così intenso in lui, di interrogarsi sul proprio lavoro, la capacità di portare a chiarezza e di definire le questioni che lo assillano. Bisogna riconoscere che il critico di Pavese è oggi molto agevolato nella sua attività da questa lucida autoconsapevolezza del suo autore. Anche se a volte è proprio l'assidua autoanalisi di Pavese a rendere piuttosto intricate e ardue certe questioni. L'ermeneutica che ha per oggetto Pavese è in buona misura opera dello stesso Pavese. Sappiamo tutti che, a partire da Goethe, da Leopardi e da Baudelaire, l'età moderna è l'età delle poetiche, ossia l'età in cui la letteratura mostra un incessante bisogno di autocontrollo critico e di autoverifica teorica. Pavese, dunque, non costituisce un'eccezione. Ma nell'Italia del primo cinquantennio del Novecento probabilmente nessun altro scrittore ha scrutato se stesso e la propria opera con un altrettanto acuto bisogno di verità. Non intendo dire che i referti che Pavese fornisce siano sempre limpidi, pertinenti e persuasivi. Dico che le sue domande non sono mai irrilevanti e che in certi casi fanno organicamente parte della sua opera letteraria con la stessa dignità che possiedono, poniamo, Lavorare stanca o Feria d'agosto o Dialoghi con Leucò.

Ciò premesso, esaminiamo rapidamente alcuni luoghi sintomatici della poetica pavesiana.

È opportuno ricordare, inizialmente, che questa poetica ruota intorno all'idea di «poesia-racconto», che, nel Mestiere di poeta, Pavese definisce per via negativa in questo modo:

Il mio gusto voleva confusamente un'espressione essenziale di fatti essenziali, ma non la solita astrazione introspettiva, espressa in quel linguaggio, perché libresco, allusivo, che troppo gratuitamente posa a essenziale (Pavese 1998: 106).

Difficilmente l'attacco alla lirica ermetica sarebbe potuto essere più chiaro e diretto. Parlare di "poesia-racconto" voleva dire innanzitutto rifiutare il genere liri- 
co, rifiutare, cioè, il genere allora dominante in Italia, con le conseguenze negative che potevano derivarne sul piano della ricezione e che ancora oggi, in qualche caso tutt'altro che trascurabile, il lavoro poetico di Pavese quasi incredibilmente è costretto a scontare. Il fatto è che in Italia molte, troppe volte la poesia è stata ed è identificata con la lirica, cosicché chi non ha coltivato la lirica non è poeta. La passione per la lirica è tale che perfino un maestro della critica quale fu Gianfranco Contini, parlando di un narratore come Gadda, ha sostenuto che "l'arte di Gadda è tutta lirica". Del resto lo stesso Contini ha negato che si possa «tenere la Commedia [di Dante] per opera narrativa» (Contini 1970: 398).

Ritornando a Pavese, è interessante che egli precisi la propria poetica dichiarando di vedere «la salvezza unicamente nell'aderenza serrata, gelosa, appassionata all'oggetto» e discorra quindi di stile oggettivo e di una «esigenza di virile oggettività nel racconto» (Pavese 1998:108-110). Strettamente congiunta con la poetica dell'«oggetto» è la poetica dell'«immagine». Pavese infatti non intende «l'immagine» come ornamento o come sovrabbondanza della rappresentazione, la percepisce come «concretezza», come «totalitaria realtà fantastica» (ibid.:111). I termini che egli adopera, se non contradittori, sono approssimativi, ma il senso che vuole comunicare non lascia dubbi. Del resto, non occorre una lunga riflessione per rendersi conto che una poetica della «poesia-racconto» e dell' «oggettività» non poteva trovare il proprio complemento e la propria chiarificazione che in una poetica dell'«immagine», cioè in un'idea di poesia fondata sulla saldezza e sulla precisione del modo di dar figura ai personaggi e rilievo alle loro vicende. Pavese non è un naturalista, anche se in certi luoghi egli riconosce di esserlo, non c'è dubbio però che abbia fatto propria la parte più fertile della lezione naturalistica.

Poiché, d'altro canto, quasi tutti i narratori, in prosa e in versi, da Dante a Verga, da Balzac a Dostoevskij, sono vivamente partecipi degli aspetti morali delle vicende che raccontano, non c'è da stupirsi se a un certo momento Pavese afferma che:

Condizione di ogni slancio di poesia, comunque alto, è sempre un attento riferimento alle esigenze etiche, e naturalmente anche pratiche, dell'ambiente che si vive (Pavese 1998: 108).

Nuovo appare però, nel contesto occidentale, quel richiamo alle «esigenze pratiche». Che non è il consueto rinvio alle aspettative del lettore ma l'impegno ad assumere pienamente nella propria opera l'«ambiente che si vive». Questa formula, dell'«ambiente che si vive», non è soltanto linguisticamente felice, è anche del tutto aderente a certe scelte tematiche e verbali compiute da Pavese nelle sue poesie. Un'ultima annotazione, prima di passare alla metrica. Pavese, a proposito del proprio lavoro, parla a volte di «naturalismo». Nel saggio A proposito di certe poesie non ancora scritte quello che appare particolarmente interessante è il rifiuto programmatico del naturalismo e la scelta o meglio la speranza di quella che lo scrittore chiama «realtà simbolica». Siamo nel 1940 e Pavese sta procedendo alla revisione e all'accrescimento di Lavorare stanca, che uscirà in seconda edizione nel 1943. Occorre chiedersi se questa idea di "realtà simbolica" abbia in qualche modo influi- 
to su quella revisione e su quell'accrescimento, e in quale rapporto essa sia con l'idea di mito.

Ma occupiamoci, per ora, di metrica. Sebbene piuttosto elementare e generico il discorso imbastito da Pavese appare sufficientemente chiaro.

Nei metri tradizionali - egli sostiene - non avevo fiducia, per quel tanto di trito e di gratuitamente [...] cincischiato ch'essi portano con sé... (Pavese 1998: 109).

Provava - aggiunge (ibid.: 110) - un «bisogno [...] di righe lunghe».

Alla genericità di questi enunciati teorici corrispondono, sul piano della prassi poetica, un totale disinteresse per il metro e il concentrarsi invece dell'attenzione sul ritmo. A Pavese non interessano né le regole né l'infrazione delle regole, quel che gli preme è raccontare ritmicamente le vicende dei suoi personaggi, modulare la narrazione secondo cadenze e inflessioni che abbiano una particolare sonorità, un timbro nettamente delineato. L'andatura ritmica risulta poi del tutto omogenea, quasi ripetitiva. Volendo usare un verbo in apparenza non pertinente, ma adoperato dallo stesso Pavese nel Mestiere di vivere e nel Mestiere di poeta, si potrebbe dire che egli tende a "mugolare" i propri racconti in versi, a convertirli in una struttura musicale che, se non fosse ben scandita, sarebbe una sorta di nenia o di cantilena. Se però si tenta un'analisi metrica delle sue poesie, ci si accorge che sotto quello che egli chiama "mugolìo ritmico", pur non volute e non cercate esistono precise misure metriche. Come ha osservato Bàrberi Squarotti, il verso è sempre suddiviso in due parti separate da una forte cesura. Addentrandosi nell'analisi, ci si avvede che il primo emistichio è quasi sempre un settenario, il secondo un settenario o un senario o un novenario, comunque una misura variabile. Giudicando secondo un criterio diverso, si può concordare con Di Girolamo, quando parla di «ritmo anapestico». Detto più semplicemente, quella su cui si regge la poesia di Pavese è una struttura trisillabica, simile a quella dei componimenti del primo Palazzeschi, autore di I cavalli bianchi, del 1905, e di Lanterna, del 1907.

Camminiàmo una séra sul fiànco di un còlle, in silènzio. Nell'ómbra del tàrdo crepùscolo mio cugìno è un gigànte vestìto di biànco, che si muòve pacàto, abbronzàto nel vólto, tacitùrno. Tacére è la nòstra virtù.

Per quanto riguarda l'assetto metrico della sua poesia, insomma, Pavese non è né un anarchico né un improvvisatore. È vero, non ubbidisce a regole metriche fisse e precise, ma aderisce a una legge fisiologica, in quanto costruisce il verso secondo l'orecchio. Si badi, costruire il verso secondo l'orecchio non vuol dire abbandonarsi a un'invenzione anomala e superficiale, al contrario significa tener conto di alcuni principi fondamentali. Nel 1950 il poeta e critico americano Charles Olson pubblica nella rivista «Poetry New York» il saggio Projective Verse, che, nel febbraio del 1961, Luciano Anceschi fa apparire tradotto in italiano nella sua rivista Il Verri. Ecco come Olson definisce il «verso proiettivo»: 
[...] L'unico verso valido sarà quello in cui il poeta riuscirà a registrare le acquisizioni del proprio udito e le pressioni del proprio respiro. [...] La sillaba è la regina [...] È dalla unione della mente con l'orecchio che è nata la sillaba. [...] E il verso [...] viene dal respiro, dalla respirazione dell'uomo che scrive, nel momento in cui scrive... (Olson 1961: 12-14).

Nello stesso fascicolo del Verri, Alfredo Giuliani - il poeta e critico che due anni dopo coordinerà e darà alle stampe la fondamentale antologia di poesia nuova $I$ Novissimi -, Alfredo Giuliani osserva:

È importante, più che non sembri, l'osservazione semplificatrice di Olson che il verso nuovo va scritto nella misura del respiro, e non per l'occhio ma secondo l'orecchio. Nonostante tutto, noi rischiamo ancora di scrivere per l'occhio... (Giuliani 1961: 52).

Nel 1935 Pavese non possedeva certo la consapevolezza critica e teorica di cui disporranno alcuni decenni dopo Olson, Giuliani e i Novissimi. A me sembra tuttavia che, in Lavorare stanca, a suo modo egli abbia costruito con notevole efficacia, se non un vero e proprio «verso proiettivo», certo un verso «secondo l'orecchio». Non è un verso sempre ritmicamente perfetto, ma corrisponde perfettamente all'esigenza di elaborare una poesia-racconto, e in pieno dominio ermetico, quando cioè a prevalere sono la lirica, l'endecasillabo e i versi brevi, esso ha probabilmente costituito un utile esempio per i poeti più giovani che agli ermetici intendevano contrapporsi. Tacendo di altre ragioni, non mi spiegherei altrimenti il fatto che Giuliani, nel suo saggio sul Verri del 1961, passando in rassegna alcuni modelli metrici si sia soffermato con particolare interesse sul modello offerto da Pavese; e che mentre antologisti e storiografi hanno quasi sempre ignorato o marginalizzato il Pavese poeta, Edoardo Sanguineti, il maggiore dei Novissimi, formatosi come Pavese a Torino, lo abbia incluso con parole di elogio nella sua antologia della poesia italiana del Novecento pubblicata nel 1969.

Veniamo alla teoria del mito. Per Pavese, carattere essenziale del mito è la sua «unicità»: il mito, egli dice, è:

[...] lo schema di un fatto avvenuto una volta per tutte, e trae il suo valore da questa unicità assoluta che lo solleva fuori del tempo e lo consacra rivelazione. Per questo esso avviene sempre alle origini, come nell'infanzia.... (Pavese 2002: 127).

Sappiamo bene che a riconoscere nell'infanzia un'età fervida di invenzioni mitiche avevano già provveduto sia Giambattista Vico, il quale diceva che «Ne' fanciulli è [...] vivida all'eccesso la fantasia» (Vico 1983: 259), sia Giacomo Leopardi, segnatamente il Leopardi del Discorso di un italiano intorno alla poesia romantica, che attribuiva ai fanciulli la capacità di compiere una «sterminata operazione della fantasia» (Leopardi 1988: 19). Per entrambi, quindi, la fanciullezza era l'età che meglio simboleggiava la condizione alla poesia. Si potrebbe pensare che Pavese, riprendendo certi temi, non potesse che ripetere le tesi dei due suoi grandi predecessori. Ma il suo per così dire "venir dopo", e avere pertanto più materiali su cui riflettere 
e una maggiore distanza temporale da cui esaminarli, consentono invece a Pavese non solo di non essere banalmente ripetitivo ma di proporre un'idea del mito e della poesia che non è priva di una sua originalità. In primo luogo, egli osserva, la poesia non si identifica con il mito, perché il mito è naturale, spontaneo, mentre la poesia «sa d'inventare». La poesia, insomma, si nutre del mito, ma possiede, o meglio è costretta a possedere una consapevolezza di sé che il mito invece non possiede. In secondo luogo Pavese distingue il mito dalla coscienza mitica. Questa coscienza subentra solamente nell'età adulta, giacché l'infanzia non sa di vivere in un tempo mitico. Dice Pavese: «le cose si scoprono, si battezzano, soltanto attraverso i ricordi che se ne hanno» (Pavese 2002: 129). E in un risvolto di copertina dichiara: «Solamente l'uomo fatto sa essere ragazzo» (ibid.: 840). Sono dunque i ricordi che consentono di passare dal mito infantile alla poesia. Certo, era stato Leopardi a inaugurare una poetica dei ricordi, ma mentre per Leopardi i ricordi e la poesia facevano rivivere un'età già di per sé mitica, anzi, la sola età mitica di cui gli esseri umani possono disporre, per Pavese sono i ricordi e la poesia che fanno transitare da un mondo mitico embrionale e potenziale a un mondo mitico pienamente attuale. La più interessante scoperta di Pavese mitologo e estetologo sta qui, nel rendersi conto che la vera età mitica e poetica non è l'infanzia, ma è l'età adulta rammemorante. L'infanzia è un deposito, un magazzino di inespresse emozioni e di esperienze folgoranti e sbalorditive che colpiscono profondamente i fanciulli ma non consentono loro raffronti, giudizi e cognizione, cosicché essi, come diceva Vico, «sentono senz'avvertire» (Vico 1983: 259). Sono gli esseri umani adulti che, recuperando quel tesoro di eventi infantili, ne scoprono l'infinita dolcezza e la stupefacente verità. Il paradosso esistenziale ed estetico è in ciò, che nella mente dell'essere umano l'esperienza diventa prodigio quando sono venute meno le condizioni che trasformavano un fatto semplicemente vitale in un fatto potenzialmente prodigioso. Pavese non procede oltre per questa strada, ma se avesse voluto trarre tutte le conseguenze dalla propria 'scoperta' sarebbe stato costretto a rendersi pienamente conto dell' $i$ nattualità e quindi dell'irrealtà che sono alla base della produzione poetica.

Un'ultima osservazione. A un certo punto del saggio intitolato L'adolescenza troviamo una frase come la seguente: «i giorni della nostra infanzia-adolescenza» (Pavese 2002: 137). È solo un frammento, ma è un frammento assai significativo, giacché ci introduce nel cuore della poetica pavesiana. La stagione potenzialmente mitica e poetica non è soltanto l'età infantile, è anche l'età adolescenziale. Ogni distanza biologica e cronologica viene meno, ogni differenza di stato e di consapevolezza risulta abolita, infanzia e adolescenza si equivalgono, anzi costituiscono la medesima unità di tempo giacché a renderle identiche provvede la loro uguale disponibilità a convertirsi in attualità mitica.

Queste, molto sommariamente riassunte e analizzate, le idee di Pavese sul mito e sulla poesia. Quando e come questa poetica del mito si converta da proposta teorica in poetica attuata sembrerebbe agevole stabilire. I tre studi estetico-antropologici cui ci siamo riferiti sono, infatti, come abbiamo detto, del 1945. Nello stesso 1945 Pavese incomincia a comporre i Dialoghi con Leucò, che usciranno nel 1947. Conviene però chiedersi: al di là di certi aspetti evidenti e tuttavia non sostanziali, $\mathrm{i}$ Dialoghi con Leucò costituiscono davvero l'attuazione della poetica delineata da 
Pavese sul piano dottrinale? Il primo a nutrire dei dubbi è lo stesso Pavese. Nel Mestiere di vivere, in data 20 febbraio 1946, leggiamo le seguenti parole:

Potendo, si sarebbe volentieri fatto a meno di tanta mitologia. Ma siamo convinti che il mito è un linguaggio, un mezzo espressivo [...] Qui ci siamo accontentati di servirci di miti ellenici data la perdonabile voga popolare di questi miti, la loro immediata e tradizionale accettabilità. Noi abbiamo orrore di tutto ciò che è incomposto, eteroclito, accidentale, e cerchiamo - anche materialmente - di limitarci, di darci una cornice [...] Non abbiamo nulla in comune coi viaggiatori, gli sperimentatori, gli avventurieri (Pavese 1952: 308).

Facciamo attenzione. È vero che questo brano diventerà 1' «Avvertenza» premessa ai Dialoghi con Leucò e che pertanto in esso lo scrittore manifesta, almeno fino a un certo segno, un punto di vista personale. Occorre però chiedersi: come mai Pavese, che nel diario, per parlare di sé, di solito adopera la seconda persona singolare, qui adopera invece la seconda plurale? Molto probabilmente egli intende riferirsi non solo a se stesso, ma anche alla sensibilità, alla mentalità, alla cultura italiana in generale. Non per nulla, sempre nel diario, poco più di un anno dopo, e precisamente alla data 10 luglio 1947, egli così si esprime, usando, questa volta, la seconda persona singolare:

Notato che Paesi tuoi e Dialoghi con Leucò nascono dal vagheggiamento del selvaggio - la campagna e il titanismo. [...] Tu vagheggi la campagna, il titanismo il selvaggio - ma apprezzi il buon senso, la misura [...] Il selvaggio t'interessa come mistero, non come brutalità storica. Non ti piacciono le storie partigiane 0 terroristiche, sono troppo spiegabili. Selvaggio vuol dire mistero, possibilità aperta. [...] Il selvaggio, il titanico, il brutale, il reazionario sono superati dal cittadino, dall'olimpico, dal progressivo (cfr. Paesi tuoi, Dialoghi con Leucò Il compagno). Tu esalti l'ordine descrivendo il disordine (Pavese 1952: 334).

Pavese è lucidamente consapevole delle proprie contraddizioni e le denuncia con molta chiarezza. Le sue frequentazioni etnologiche e psicoanalitiche e soprattutto la lettura di Nietzsche - che nel diario è esplicitamente citato - gli consentono di conoscere la vera natura del mito e quindi di rendersi conto dei limiti e dei difetti dei pur amati Dialoghi con Leucò.

I Dialoghi sono un unicum nella letteratura italiana contemporanea, e in quanto tali vanno tenuti da noi nella giusta considerazione. Ma sono un unicum che imprigiona il mito, lo riduce letterariamente, lo costringe in una forma elegante e rigida. Ciò che il mito ha di selvaggio e di impervio va completamente perduto. Per usare i termini di Nietzsche, nei Dialoghi il mito è trattato in modi apollinei, mentre la sua natura richiederebbe che venisse trattato in modi dionisiaci. Pavese sentiva indubbiamente il richiamo di ciò che chiamava «il selvaggio», e cioè del dionisiaco, ma la sua educazione lo costringeva a credere che soltanto se veniva addomesticato e addolcito il dionisiaco poteva diventare oggetto di un'esperienza letteraria. I Dialoghi con Leucò sono l'opera di uno scrittore indubbiamente originale, incapace però 
di liberarsi da una doppia influenza: l'influenza di Benedetto Croce e l'influenza della prosa d'arte.

«Il mito è un linguaggio» sostiene Pavese. Non vi sarebbe nulla da obiettare, se non fosse che nei Dialoghi egli sostituisce al mito la mitologia, anzi, peggio, sostituisce ai propri miti personali la mitologia greca. Né si può controbattere che la mitologia greca maschera e al tempo stesso manifesta i miti personali dello scrittore. I Dialoghi esprimono certamente una visione personale della vita, ma non attingono se non a tratti e allusivamente alla zona mitica più profonda che vive nella mente dello scrittore. Rivelatore, da questo punto di vista, è il dialogo intitolato $L a$ belva, dove il vero tema è il «selvaggio», ma dove il «selvaggio» è appena nominato e ad esso soltanto si allude. Anzi, non si allude. Del «selvaggio» si parla parlando d'altro. Si scopre così la vera natura dei Dialoghi con Leucò. La mitologia è soltanto una superficie, una buccia, una scorza. In realtà I Dialoghi sono un libro di allegorie. E noi sappiamo bene che cos'è un'allegoria. Ce lo hanno insegnato vari autori, da Quintiliano a Walter Benjamin. Allegoria, spiega Benjamin, vuol dire che «Ogni personaggio, qualsivoglia cosa, qualsiasi situazione possono significarne un'altra qualunque» (Benjamin 1971: 184). Pavese non voleva e probabilmente non poteva dire immediatamente e direttamente l'orrore che provava. E lo ha espresso allora mediante delle allegorie.

Peraltro (se si esclude, come vedremo, Il dio-caprone) ciò che egli chiama il «selvaggio» non fa propriamente parte dei miti di Pavese. Come attesta il breve frammento incompiuto intitolato $\mathrm{Il} \mathrm{sangue}^{l}$, egli è attratto dall'orrore, ma l'orrore, per lui, piuttosto che un mito, è un'ossessione. I veri miti di Pavese sono legati all'infanzia e all'adolescenza (e all'eros, o meglio al sesso) e pertanto, se vogliamo trovare un'attuazione della poetica teorica affidata ai tre saggi estetico-antropologici, doppiamo cercarli nei racconti di Feria d'agosto, tanto più che questi racconti precedono di poco quei saggi e sono raccolti dall'autore nel medesimo volume.

Nella carriera di Cesare Pavese c'è un mito che precede non soltanto la teoria del 1940 ma anche la consapevolezza che con gli anni lo scrittore verrà acquisendo soprattutto grazie alla lettura delle opere di etnologia. È il mito che ispira certe poesie di Lavorare stanca, un mito la cui presenza è agevole avvertire ma di cui non è facile definire i caratteri. Vissuto in un certo modo, questo mito avrebbe potuto sia accentuare, aggravandoli, il lirismo e l'autobiografismo che caratterizzavano il lavoro poetico antecedente a Lavorare stanca, sia annullare o attenuare lo guardo realistico che l'autore rivolge alle cose. Quello che avviene è invece abbastanza sorprendente: senza cancellare il soggetto, e senza abolire la ruvida concretezza di ciò che i sensi percepiscono e l'immaginazione propone, il ricorso al mito amplia ed espande l'attenzione così da investire una pluralità di personaggi e di oggetti, mentre al tempo stesso rende più netto e visibile il rilievo di ciò che appare. Tutto questo premesso, occorre, a mio avviso, guardarsi da un rischio che Marziano Guglielminetti ci ha aiutato a evitare quando ha dichiarato che «l'«impurità» è la chiave di lettura migliore di Lavorare

\footnotetext{
1 «Il mio orrore del sangue...». Il tema del sangue era già presente in Il dio-caprone. Un testo nel quale però è assente l'orrore ed è invece evidente quanto Pavese fosse ossessionato dal sesso.
} 
stanca» (Guglielminetti 1998: xi). Il rischio, a mio modo di vedere, è quello di credere che i componimenti che costituiscono Lavorare stanca siano tutti di ispirazione mitica. «Impurità», a me sembra, oltre che «rappresentazione non selezionata della realtà», come suggerisce Guglielminetti (ibid.), cioè assenza della "purezza" ermetica, vuol dire anche alternanza di ispirazione e quindi alternanza stilistica. Cosicché, se non ci possono essere dubbi sul carattere mitico di testi come Il dio-caprone, o come Paternità, o come Mito, non ci possono essere dubbi neppure sul realismo di poesie come Proprietari o come Casa in costruzione:
Al levar della luna le capre non stanno più chete, ma bisogna raccoglierle e spingerle a casa, altrimenti si drizza il caprone. Saltando nel prato sventra tutte le capre e scompare. Ragazze in calore dentro i boschi ci vengono sole, di notte, e il caprone, se belano stese nell'erba, le corre a trovare. Ma, che spunti la luna: si drizza e le sventra.
E le cagne, che abbaiano sotto la luna, è perché hanno sentito il caprone che salta sulle cime dei colli e annusato l'odore del sangue.
E le bestie si scuotono dentro le stalle.
Solamente i cagnacci più forti dan morsi alla corda E qualcuno si libera e corre a seguire il caprone, che li spruzza e ubriaca di un sangue più rosso del fuoco, e poi ballano tutti, tenendosi ritti e ululando alla luna.

(Il dio-caprone)

Qui, e non nei Dialoghi con Leucò, è il vero mito di Pavese. Un mito dionisiaco, pervaso dall' «odore del sangue», che, si tratti di «ragazze in calore» o di bestie, non sublima la vita sessuale ma, al contrario, ne esibisce l'aspetto animalesco. Facendo probabilmente inorridire non solo i censori fascisti ma anche i poeti ermetici. Leggiamo però anche, quasi in un 'impuro' controcanto, almeno la parte iniziale di Proprietari:

Il mio prete che è nato in campagna, è vissuto vegliando giorno e notte in città i moribondi e ha riunito in tanti anni qualche soldo di lasciti per l'ospedale.

Risparmiava soltanto le donne perdute e i bambini e nel nuovo ospedale - lettucci di ferro imbiancato c'è un'intera sezione per donne e bambini perduti. Ma i morenti che sono scampati, lo vengono ancora a trovare E gli chiedon consigli di affari. Lo zelo lo ha reso ben magro tra il sentore dei letti e i discorsi con gente che rantola e seguire, ogni volta che ha tempo, i suoi morti alla fossa e pregare per loro, spruzzandoli e benedicendoli.

Nessun sentimento religioso, nessuna ammirazione per il prete zelante, nessuna pietà per i moribondi. Eppure non siamo di fronte $\mathrm{a}$ un freddo resoconto. Sono quel- 
le che forse potremmo chiamare curiosità intellettuale e sociale e passione di raccontare ad animare e a rendere vivace, qui e altrove, la rappresentazione.

Per la verità, se ci fosse il tempo di addentrarsi nell'analisi, il discorso dovrebbe essere meno reciso e rilevare che, accanto a componimenti che, a occhio nudo, si rivelano nettamente contrassegnati da un determinato carattere, stanno componimenti in cui visione realistica e visione mitica coesistono o meglio ancora si fondono. Tale è la poesia più famosa del libro di Pavese, e cioè I mari del Sud. Anche questa, se vogliamo, è una specie di «impurità».

L' «impurità» individuata da Guglielminetti in Lavorare stanca consente una lettura aderente del libro. Il problema è stabilire se l' «impurità» coincida con quella varietà o pluralità tematica che stava a cuore a Pavese o se sia invece altra cosa. Incominciamo con l'osservare che Lavorare stanca è, in primo luogo, il racconto di un'educazione sentimentale. Soggetto di questa educazione è il ragazzo protagonista o coprotagonista di poesie come I mari del Sud e Antenati. Ma l'«impurità» investe già questo soggetto, perché a volte esso si identifica con la prima persona, come accade appunto in I mari del sud e in Antenati, altre volte coincide invece con la terza persona, come succede in Città in campagna o in Civiltà antica o in Esterno:

Il ragazzo respira più fresco, nascosto dalle imposte, fissando la strada. Si vedono i ciottoli per la chiara fessura, nel sole. Nessuno cammina per la strada. Il ragazzo vorrebbe uscir fuori così nudo - la strada è di tutti - e affogare nel sole.

In città, non si può. Si potrebbe in campagna, se non fosse, sul capo, il profondo del cielo che atterrisce e avvilisce. C'è l'erba che fredda fa il solletico ai piedi, ma le piante che guardano ferme, e i tronchi e i cespugli son occhi severi per un debole corpo slavato, che trema.

Fino l'erba è diversa e ripugna al contatto.

(Civiltà antica)

Non basta, occorre infatti tener conto sia del personaggio del giovane adulto, che compare in molti testi e in cui non è difficile riconoscere il ragazzo cresciuto; sia, infine, di personaggi diversi tanto dal ragazzo quanto dall'adulto, come l'«eremita» di Paesaggio I, o come il «vecchio», o come le varie donne. A proposito di queste, conviene osservare che in una poesia come Gente che non capisce quella che viene rappresentata è una vera e propria educazione sentimentale della protagonista, Gella:

Sotto gli alberi della stazione si accendono i lumi.

Gella sa che a quest'ora sua madre ritorna dai prati

col grembiale rigonfio. In attesa del treno

Gella guarda tra il verde e sorride al pensiero

di fermarsi anche lei, tra i fanali, a raccogliere l'erba. 
Gella sa che sua madre da giovane è stata in città una volta: lei tutte le sere col buio ne parte e sul treno ricorda vetrine specchianti e persone che passano e non guardano in faccia. La città di sua madre è un cortile rinchiuso tra muraglie e la gente s'affaccia ai balconi. Gella torna ogni sera con gli occhi distratti di colori e di voglie, e, spaziando dal treno, pensa, al ritmo monotono, netti profili di vie tra le luci, e colline percorse di viali e di vita e gaiezze di giovani, schietti nel passo e nel riso padrone.

Basta la lettura di queste due prime strofe per accorgersi che forse Pavese non ha mai raggiunto un persuasivo risultato di poesia usando mezzi così semplici e così lontani dalla "Poesia". E per rendersi conto che l'ipotesi del racconto di una educazione sentimentale va estesa a diversi personaggi, ma può estendersi soltanto se la si riferisce in primo luogo al narratore. È il narratore che compie la propria educazione inventando certi personaggi e certe loro vicende. Del resto nessuno può escludere che quando un poeta o un romanziere, da Dante a Flaubert, compie il resoconto di un'educazione sentimentale egli educhi anche o in primo luogo se stesso. Sperimentando il proprio linguaggio egli al tempo stesso mette alla prova e incrementa la propria esperienza di vita. Per quanto mi riguarda, non conosco un'autoeducazione sentimentale più intensa e più rivelatrice di quella di cui Dante si rende l'oggetto attraverso la confessione di Francesca nel canto V dell'Inferno.

Dobbiamo, in ogni caso, cercare di definire il mito in Lavorare stanca. Incominciando con l'osservare che, più che all'invenzione tematica, esso è legato alla scansione ritmica. Come aveva capito perfettamente Carlo Dionisotti, c'è nel libro un «ritmo di epopea e di leggenda» che conferisce agli oggetti un'aura favolosa, e sia pure di favola agreste, contadina (Dionisotti 1945: 5). E, insomma, soprattutto il particolare modo di raccontare di Pavese che inserisce le cose in un orizzonte di quotidianità che, in certe poesie, acquista immediatamente le forme e i colori di un mondo fantastico. Se si leggono con attenzione certe pagine del Mestiere di vivere datate 1936, lo stesso anno, cioè, in cui appare Lavorare stanca, ci si rende subito conto che Pavese ha guardato e guarda ad Omero come ad un modello. Egli dice, per esempio: «È bene rifarsi a Omero» (Pavese 1952). Poi, fermando l'attenzione sull' unità materiale dei personaggi omerici, pone una questione che doveva sembrargli fondamentale (ibid.):

Ora si apre il problema se, in poesie separate non sia possibile rifare il miracolo; non per altra ragione che tendendo sempre la mente all'unità in tutte le sue manifestazioni. Comporre secondo l'estro, ma con sotterranea abilità far concorrere i vari pezzi a un poema (Pavese 1952: 212).

Scrivere un poema vero e proprio - ragiona Pavese - oggi non è più possibile. Si tratta di vedere, invece, se componendo «poesie separate» non sia possibile collegarle fra loro in modo da farle apparire un «poema». La riflessione, si badi, non si 
distacca da Omero. Dice ancora Pavese: «Più ci penso e più mi appare notevole il fare omerico del libro-unità» (ibid.). Pur trascurato o ignorato, Pavese ha portato un notevole contributo al rinnovamento della poesia in Italia. Un contributo che non mancherà di risultare fruttuoso nelle generazioni successive, quando, con i Novissimi, il compito sarà quello di liquidare l'esperienza lirica per conquistare modi più liberi e aperti e vicini alla lingua parlata e, per esempio, in qualche poesia di Sanguineti risuonerà l'eco di certe scansioni pavesiane. Riconosciuto questo, occorre riconoscere anche che le riflessioni del Mestiere di vivere su cui ci siamo soffermati mostrano che Pavese è tutto nella tradizione, ma anche che la sua tradizione non è quella della maggior parte dei poeti suoi contemporanei, italiani e stranieri. D'altro canto, come è ben comprensibile, Omero non è e non può essere il suo unico modello. A parte quelli che sono stati da tempo individuati dagli studiosi, in primo luogo Whitman, ci sono anche coloro che Pavese definisce i «consorti» di Omero, cioè Dante, e, in misura minore, $\mathrm{o}$, meglio, in modi diversi, Shakespeare e Baudelaire, ossia quelli che egli definisce «favolosi, potenti costruttori che si deliziano del particolare, sentito fino allo svolazzo, che respirano tutta la vita a regolari e perfetti respiri quotidiani» (Pavese 1952: 156). Può apparire singolare che Pavese si interroghi intorno al problema dell'unità e della varietà del libro di poesie proprio adesso che ha concluso la prima stesura di Lavorare stanca, ma, anche se non ne abbiamo testimonianza scritta, è molto probabile che egli abbia affrontato simili questioni già durante la composizione del libro, se non precedentemente. Non dobbiamo inoltre dimenticare che nel 1936 Lavorare stanca, sia prima sia dopo l'uscita della prima edizione, è ancora un work in progress e che quindi il suo autore ha buoni motivi per porsi certe domande.

E chiaro, in ogni caso, che Pavese non aspirava ad essere un Omero moderno. Omero e Dante sono per lui semplici modelli strutturali, non paradigmi etici ed estetici da frequentare con venerazione e da imitare con la maggiore fedeltà possibile. Profondamente diversi l'uno dall'altro, essi possono essere accomunati solo sulla base della nozione di «poesia-racconto», cioè in quanto consentono di lasciarsi alle spalle il consunto modello lirico-autobiografico e obbligano a un certo grado di oggettivazione e quindi alla creazione di personaggi. La «poesia-racconto» è rara nella letteratura italiana del Novecento, ma non è rara nella letteratura moderna occidentale. Mentre è raro che un autore di «poesia-racconto» assuma come modelli strutturali l'Iliade, l'Odissea e la Commedia. Voglio dire che la presenza del mito in Lavorare stanca è imposta anche da quei modelli, che richiedono sì l'unità nella varietà, ma sollecitano anche a scegliere un certo modo di trattare gli oggetti, una certa tonalità. Un modo, una tonalità che potremmo chiamare 'mitici', cioè capaci di proporre l'oggetto emozionalmente e di conferirgli nettezza e risalto come se l'oggetto si manifestasse in una sorta di alba della visione o si costituisse come un'apparizione insieme naturale e fantastica. Si pensi a quel personaggio, poeticamente davvero gigantesco, portato in luce, in I mari del Sud, da questi versi: «Nell'ombra del tardo crepuscolo / mio cugino è un gigante vestito di bianco / che si muove pacato, abbronzato nel volto, / taciturno». Versi di cui non ci si può limitare a dire che proiettano una luce di mito su tutto il componimento, giacché quasi l'intero testo, e segnatamente la parte conclusiva, sono sollecitati da una sorta di vibrazione mitica: 


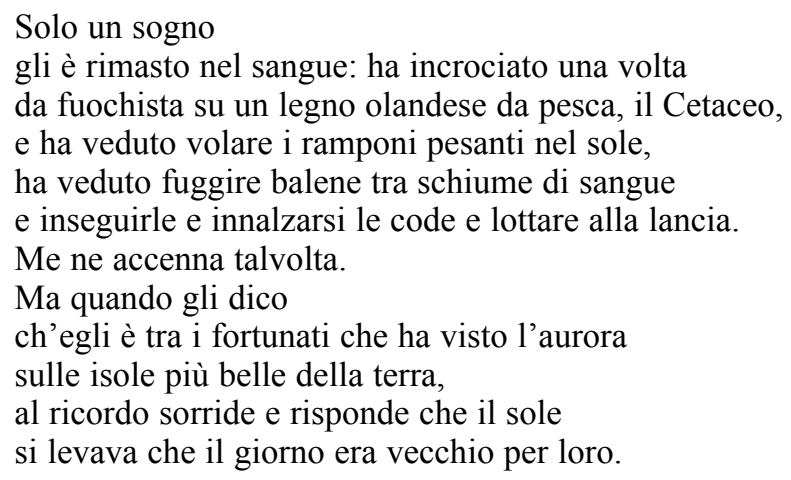

Senza peraltro che, a metà del testo, Pavese rinunci al suo minuto realismo di narratore in versi tentato dalla prosa:

Mio cugino ha una faccia recisa. Comprò un pianterreno nel paese e ci fece riuscire un garage di cemento con dinanzi fiammante la pila per dar la benzina e sul ponte ben grossa alla curva una targa-réclame. Poi ci mise un meccanico dentro a ricevere i soldi e lui girò tutte le Langhe fumando.

Oppure si pensi ai versi che chiudono la poesia Antenati:

Ho trovato una terra trovando i compagni, una terra cattiva, dov'è un privilegio non far nulla, pensando al futuro.

Perché il solo lavoro non basta a me e ai miei, noi sappiamo schiantarci, ma il sogno più grande dei miei padri fu sempre un far nulla da bravi. Siamo nati per girovagare su quelle colline, senza donne e le mani tenercele dietro alla schiena.

I mari del Sud e Antenati inaugurano Lavorare stanca e danno il tono, se non a tutto il libro, a molte composizioni fra le più significative. Questi sono i nuovi eroi e le nuove eroine della moderna epica: contadini, contadine, prostitute, vecchi, ragazzi. Se siamo disposti ad adoperare categorie flaubertiane, possiamo dire che, abbandonato il «sublime d'en haut» di Omero e di Dante, non più usufruibile anche da chi, a suo modo, aspiri a creare un poema, Pavese assuma un suo «sublime d'en bas», che gli serve a dar vita a un mondo di personaggi medio-bassi, sprovvisti di coturni tragici e della protezione di divinità ma non privi del rilievo che conferiscono loro la simpatia e la capacità verbale del loro autore. Che questi personaggi siano spesso socialmente o psichicamente anomali non solo non può stupire ma corrisponde alle regole non scritte di chi intende fondare un moderno epos borghese-contadino, anzi un anti-epos che egli preferisce chiamare «poesia-racconto». Pavese non contesta gli ermetici, si limita a ignorarli, perché il suo problema è un altro: con- 
testare o meglio mettere in questione l'epos usando modi che sono propri dell'epos. Meglio ancora, la contestazione più efficace della lirica ermetica sta per Pavese nella creazione di un'epica borghese-contadina che risulti congrua alla sensibilità e alla cultura della società moderna.

Certo, conseguenze operative diverse da quelle costituite da Lavorare stanca si sarebbero avute se Pavese avesse scelto modelli strutturali diversi, l'Orlando furioso, poniamo. Dove l'unità nella varietà raggiunge effetti sorprendenti ma non esclude un'ironia sorridente che non corrisponde certo alla sensibilità di Pavese. Pavese non lo dice esplicitamente, ma l'insistenza sul nome di Omero mostra che per lui la «poesia-racconto» deve prendere il posto dell'epos, non può che essere l'unica forma di epos che l'età moderna consente allo scrittore. Del resto, anche se probabilmente Pavese non lo sapeva, in una bellissima pagina dell'Estetica Hegel sostiene che il romanzo è «la moderna epopea borghese» (Hegel 1967: 1223). A questo punto mi guardo bene dal pensare che Lavorare stanca sia soltanto un tentativo, un'approssimazione, un proto-micro-romanzo sperimentale in versi, cui dovranno seguire prove più mature in prosa, cioè dei romanzi veri e propri. Lavorare stanca ha una piena autonomia e una piena compiutezza espressiva, ed esercita una funzione fondamentale non soltanto nel contesto italiano, giacché mostra esemplarmente che in un'età che sembrerebbe ammettere soltanto la poesia lirica è invece possibile dare forma poetica a un'autentica vocazione narrativa, creare cioè un moderno epos.

\section{Bibliografia}

BENJAmin, Walter (1971): Il dramma barocco tedesco. Torino, Einaudi.

ConTINI, Gian Franco (1970): Varianti e altra linguistica. Torino, Einaudi.

DiONISOTTI, Carlo (1945): «Lavorare stanca», in La Nuova Europa, II, 34, 26 agosto 1945, p. 5.

GIULIANI, Alfredo (1961): «Il verso secondo l'orecchio», Il verri, n. 1, pp. 43-52.

GuglielminetTI, Marziano (1998): «Introduzione» a C. Pavese (1998), pp. X-xxv.

HEGEL, Georg Wilhelm Friedrich (1967): Estetica.Torino, Einaudi.

LEOPARDI, Giacomo (1988): Discorso di un italiano intorno alla poesia romantica. Bellinzona, Casagrande.

OLSON, Charles (1961): «Il verso secondo l'orecchio», Il verri, n. 1., 1961, pp. 9-23.

PAVESE, Cesare (2002): Tutti i racconti. Torino, Einaudi.

PAVESE, Cesare (2000): Il mestiere di vivere. Diario 1935-1950, edizione condotta sull'autografo a cura di Marziano Guglielminetti e Laura Nay, nuova introduzione di C. Segre. Torino, Einaudi.

PAVESE, Cesare. (1998): Le poesie. Torino, Einaudi.

VICO, Gian Battista (1983): Autobiografia, Poesie, Scienza nuova. Garzanti, Milano. 